\title{
Bundesverfassungsgericht - (Noch immer) Kein Verfassungsverstoß durch die Beschränkung der Erbringung von MRT-Leistungen auf Radiologen im GKV-System
}

\section{Vorbemerkung}

Mit der Entscheidung des Bundesverfassungsgerichts (BVerfG) vom 02.05.2018 geht eine langjährige gerichtliche Auseinandersetzung um die Frage zu Ende, ob andere Fachärzte als Radiologen zur Erbringung und Abrechnung von kernspintomographischen Leistungen im Rahmen der vertragsärztlichen Versorgung berechtigt sind. Das vorliegende Verfahren war insofern von besonderer Pikanterie, als der Kläger, ein Facharzt für Innere Medizin und Kardiologie, der Auffassung war, dass Kardiologen zur Durchführung kernspintomografischer Untersuchungen des Herzens sogar besser qualifiziert seien als alle bzw. bestimmte Ärzte für Radiologie.

Dieser Ansicht hat die AG Herz- und Gefäßdiagnostik der Deutschen Röntgengesellschaft e. V. (DRG) bereits in einer Stellungnahme vom Juni 2011 inhaltlich und fachlich ausdrücklich widersprochen (vgl. RöFo-Sonderdruck, Zum aktuellen Standort der Radiologie in der Patientenversorgung, Stellungnahme der deutschen Röntgengesellschaft, Juni 2011). Die vom BVerfG nun bestätigte Argumentation soll hier noch einmal zur Verdeutlichung widergegeben werden:

1. Die Ausbildung in kardialer Bildgebung (CT, MRT) ist integraler Bestandteil der radiologischen Weiterbildung. In der Ausbildung zum Kardiologen dagegen ist die Vermittlung von Spezialwissen auf dem Gebiet der MRT und CT des Herzens nicht enthalten.

2. Die DRG verfügt schon seit mehr als 18 Jahren über eine strukturiertes Fortbildungsprogramm und ein internes Qualifizierungs- und Qualitätssicherungssystem. Radiologen suchen in der Herzbildgebung zweifelsfrei die Interaktion mit den Kardiologen; daraus darf aber nicht abgeleitet werden, dass ein Kardiologe für die Fortbildung von Radiologen besonders geeignet oder gar erforderlich ist. Genau das Gegenteil ist der Fall: ein Kardiologe, der die Zusatz- qualifikation „MRT des Herzens“ erwerben möchte, muss nach der gültigen Rechtslage die Weiterbildung bei einem Radiologen nachweisen.

3. Der klinische Erfolg der Methode beruht in erster Linie auf technischen Weiterentwicklungen. Gerade kardiale MRT Untersuchungen stellen bereits bei der Durchführung eine besondere Herausforderung dar, weil das Protokoll und Messparameter für viele Patienten individuell angepasst werden müssen. Diese hohe technische Kompetenz in der MRT ist wesentlicher Bestandteil der radiologischen Weiterbildung und ein Alleinstellungsmerkmal radiologischer Fachärzte.

4. Die generelle Einschätzung, dass klinisches Spezialwissen in Bezug auf das untersuchte Organ, dass nur in den entsprechenden klinischen Weiterbildungen vermittelt wird, für die Interpretation radiologischer Bilder zwingend erforderlich ist, ist falsch. Vielmehr zeichnet sich die Kompetenz des Radiologen heute dadurch aus, dass sowohl klinisches Fachwissen, als auch methodische Expertise für bildgebende diagnostische verfahren in den diagnostischen Prozess einfließen und auf vielen Gebieten der Medizin in hohem Maße zur schnellen und zielgenauen Diagnostik und Therapie der Patienten beitragen.

5. Bei jeder kardialen MRT werden andere Organe (Lunge, Mediastinum, Mamma, große Gefäße, Skelett/Wirbelsäule, Oberbauch) miterfasst, die dann auch mit beurteilt werden müssen. Die umfassende Ausbildung zur Beurteilung aller Strukturen ist integraler Bestandteil der radiologischen Weiterbildung und ein weiteres Alleinstellungsmerkmal radiologischer Fachärzte.

6. Die Trennung zwischen bildgebender Diagnostik und klinischer Patientenversorgung macht auch wirtschaftlich Sinn, da sie kostentreibende Selbstzuweisungen verhindert. In der jüngst verfügbaren Übersicht des Europäischen Kardio-CT-Registers der ESC erreicht die Selbstzuweisungsrate der Kardiologen $60 \%$ und mehr.

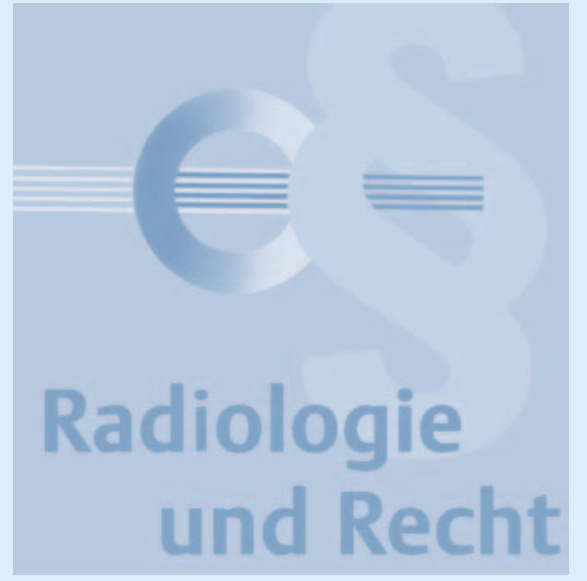

Damit ist bereits weiterbildungsrechtlich und der fachlichen Ausbildung des Radiologen widerlegt, dass Fachärzte für Innere Medizin mit dem Schwerpunkt Kardiologie über eine bessere Ausbildung und Erfahrung im Bereich der MRT-Diagnostik des Herzens verfügen. In seiner viel beachteten Stellungnahme „Zur Situation der diagnostischen Radiologie in der Bundesrepublik Deutschland (mit besonderer Berücksichtigung der MRT)“ in RöFo 170 (1999), M74, 80 hat der frühere Präsident der Deutschen Röntgengesellschaft, Herr Prof. Günther Kauffmann auf diesen Umstand bereits hingewiesen und festgestellt, dass nur eine enge Verflechtung von eingehenden Kenntnissen, Erfahrungen und Fertigkeiten sowohl in der Computertomografie als auch in der Magnetresonanztomografie „eine fach- und fallgerechte Indikationsstellung, eine optimale Durchführung und eine korrekte differentialdiagnostische Befundung im Kontext anderer bildgebender Verfahren ermöglicht“. An dieser zutreffenden Einschätzung kann nach der Entscheidung des BVerfG festgehalten werden.

\section{Einleitung}

Im vergangenen Jahr lag dem BVerfC bereits zum dritten Mal die Frage der Verfassungsmäßigkeit der Kernspintomographie-Vereinbarung ${ }^{1}$ (KernspinV) zur Entscheidung vor, nach der die Erbringung

1 Vereinbarung von Qualifikationsvoraussetzungen gemäß § 135 Abs. 2 SGB V zur Durchführung von Untersuchungen in der Kernspintomographie vom 10.02.1993, Stand 01.01.2015. 
und Abrechnung magnetresonanztomographischer (MRT) Leistungen zu Lasten der gesetzlichen Krankenversicherung (GKV) allein Radiologen ${ }^{2}$ vorbehalten ist.

Zunächst 2001 hatte ein Orthopäde gegen die Versagung einer Genehmigung zur Erbringung und Abrechnung von MRTLeistungen im GKV-System erfolglos Verfassungsbeschwerde beim BVerfG erhoben. Danach wandte sich 2007 ein Kardiologe Direktor einer Klinik für Innere Medizin/ Kardiologie und seit vielen Jahren zur Erbringung vertragsärztlicher Leistungen ermächtigt - wegen der Versagung einer solchen Genehmigung ebenfalls erfolglos an das BVerfG. Nachdem er die Zusatzqualifikation „MRT - fachgebunden -“ erworben hatte, versuchte es der Kardiologe erneut: Er klagte sich durch die Instanzen der Sozialgerichtsbarkeit und erhob 2014 abermals Verfassungsbeschwerde bei dem BVerfG. Dieses stellte in einem Nichtannahmebeschluss erneut fest, dass eine Beschränkung der Erbringung von MRT-Leistungen im GKV-System auf Radiologen (und Nuklearmediziner) nicht gegen das Grundgesetz (GG) verstößt (vgl. BVerfG, Nichtannahmebeschluss vom 02.05.2018, Az.: 1 BvR 3042/14).

\section{Hintergrund der Entscheidung}

Der Beschwerdeführer, ein Kardiologe, der nach eigener Aussage maßgeblich an der Entwicklung der Technik von MRT-Untersuchungen des Herzens beteiligt war, führte bereits seit Jahren entsprechende Untersuchungen bei privatversicherten Patienten durch. Bereits 2001 beantragte er als ermächtigter Arzt eine Genehmigung zur Erbringung und Abrechnungen von MRT-Leistungen für gesetzlich Krankenversicherte. Die zuständige Kassenärztliche Vereinigung (KV) versagte ihm jedoch die begehrte Genehmigung; die Qualifikationsanforderungen des §4 Abs. 1 der KernspinV lägen nicht vor, insbesondere weil der Kardiologe

2 Ursprünglich waren auch Nuklearmediziner zur Erbringung von MRT-Leistungen nach der Kernspintomographie-Vereinbarung berechtigt. Das Fachgebiet der Nuklearmedizin führt die MRT jedoch nicht mehr als Teil der Weiterbildungsinhalte. nicht über die Gebietsbezeichnung „Diagnostische Radiologie“ verfügte. Der gegen diese Genehmigungsversagung geführte Rechtsstreit blieb erfolglos, sodass sich der Kläger schließlich an das BVerfG wandte, das 2010 über diesen Fall entschied (vgl. BVerfG, Nichtannahmebeschluss vom 08.07.2010, Az.: 2 BvR 520/07). Den Kern der Entscheidung bildete dabei die Frage, ob der Kardiologe - trotz der nach seinen persönlichen Fähigkeiten vorliegenden Qualifikation für die Erbringung von MRTUntersuchungen des Herzens - durch die Versagung der Abrechnungsgenehmigung durch die zuständige $\mathrm{KV}$ in seiner durch Art. 12 Abs. 1 GG garantierten Berufsfreiheit verletzt war.

Das BVerfG konnte jedoch keinen Verstoß gegen Verfassungsrecht feststellen: Zwar werde durch die Versagung der Genehmigung auf Grundlage des §4 Abs. 1 KernspinV in die Berufsfreiheit des Beschwerdeführers eingegriffen, so das BVerfG. Er sei allerdings nicht in seinem Status als Arzt betroffen, da der Kardiologe nicht im Kernbereich seines Fachgebiets eingeschränkt werde. Die Beschränkung der kardiologischen Tätigkeit im GKV-System betreffe den Kardiologen nur in einem Teilausschnitt seiner ärztlichen Tätigkeit, die eigentliche Berufstätigkeit als Grundlage der Lebensführung bleibe unberührt. Eine derartige Beschränkung der Berufsausübung sei weiterhin aus Gründen des Gemeinwohls auch verfassungsrechtlich gerechtfertigt, da die Konzentration aller MRT-Leistungen bei speziell qualifizierten Ärzten der Qualität der Versorgung sowie der Wirtschaftlichkeit im Interesse der Funktionsfähigkeit der gesetzlichen Krankenversicherung diene. Zudem solle die Konzentration der MRT-Leistungen den Erhalt der diagnostisch tätigen Ärzte als Berufsgruppe gewährleisten, sodass es auf die individuelle Qualifikation des Kardiologen nicht ankäme. Damit knüpft das BVerfG unmittelbar an seine Ausführungen aus dem Jahr 2004 an, als es über die Verfassungsmäßigkeit des Ausschlusses des Orthopäden von der Erbringung von MRT-Leistungen auf Grundlage der KernspinV zu entscheiden hatte (vgl. BVerfG, Nichtannahmebeschluss vom 16.07.2004, Az.: 1 BvR 1127/01).

\section{Zusatzweiterbildung „MRT} - fachgebunden -“ berechtigt nicht zur Erbringung von MRT-Leistungen im

\section{GKV-System}

Im Jahr 2004 führte die für den klagenden Kardiologen zuständige Ärztekammer die Zusatzweiterbildung „MRT - fachgebunden -“ für die Erlangung der fachlichen Kompetenz zur Durchführung und Befundung gebietsbezogener MRT in Ergänzung zu einer Facharztkompetenz ein. Die Einführung dieser Zusatzweiterbildung ging auf den Beschluss des 106. Deutschen Ärztetages zurück, der mittlerweile in allen Bundesländern umgesetzt wurde. Kurz nach dem Beschluss des Deutschen Ärztetages wurde vor dem Hintergrund der ergangenen bundessozialgerichtlichen Entscheidung zur Versagung von MRT-Abrechnungsgenehmigungen für Orthopäden (vgl. BSG, Urteil vom 31.01.2001, Az.: B 6 KA 24/00 R) eine neue Regelung in Satz 4 des $§ 135$ Abs. 2 SGB V durch das GKV-Modernisierungsgesetz eingeführt (vgl. BT-Drs. 15/1525, S. 124). Nach $\S 135$ Abs. 2 Satz 1 und 2 SGB $\checkmark$ können die Vertragspartner der Bundesmantelverträge für Leistungen, die einer besonderen Fachkunde bedürfen, entsprechende Voraussetzungen für die Ausführung und Abrechnung dieser Leistungen in Qualitätssicherungsvereinbarungen regeln. Anforderungen für die Leistungserbringung müssen sich allerdings nach dem ärztlichen Weiterbildungsrecht richten, soweit das ärztliche Berufsrecht bundesweit inhaltsgleiche Qualifikationsvoraussetzungen für bestimmte Leistungen vorsieht.

Von diesem Grundsatz macht $§ 135$ Abs. 2 Satz 4 SGB V eine Ausnahme: Er gestattet den Vertragspartnern der Bundesmantelverträge, zur Sicherung der Qualität und Wirtschaftlichkeit der Leistungserbringung, abweichend von den Weiterbildungsordnungen des ärztlichen Berufsrechts Regelungen zu treffen, nach denen die Erbringung bestimmter medizinisch-technischer Leistungen den Fachärzten vorbehalten ist, für die diese Leistungen zum Kern ihres Fachgebietes gehören.

Nachdem dem Kardiologen von der zuständigen Ärztekammer die Berechtigung 
erteilt wurde, die Zusatzbezeichnung „MRT - fachgebunden -“ zu tragen, beantragte er im Jahre 2007 erneut die Genehmigung zur Erbringung und Abrechnung von MRTUntersuchungen des Herzens bzw. des Thorax sowie die Abrechnungsgenehmigung für Leistungen der MR-Angiographie bei der zuständigen KV. Die KV lehnte beide Anträge ab. Zur Begründung wurde angeführt, dass der Kardiologe weder die von der KernspinV geforderte Gebietsbezeichnung „Diagnostische Radiologie“, noch die von der Qualitätssicherungsvereinbarung zur MR-Angiographie 3 (MR-AngioV) geforderte Fachgebietsbezeichnung „Radiologie“ führen dürfe. Für die Erteilung der Abrechnungsgenehmigung sei allein die Zugehörigkeit zum Fachgebiet Radiologie und nicht die Zusatzbezeichnung maßgeblich. Die gegen die Versagung der Genehmigungen gerichtete Klage vor dem Sozialgericht (SG) hatte zunächst Erfolg (vgl. SG Berlin, Urteil vom 06.04.2011, Az.: S 71 KA 151/10). Das SG befand, dass wegen des in Art. 3 Abs. 1 GG verankerten Gleichheitsgrundsatzes ein Kardiologe mit der Zusatzqualifikation „MRT - fachgebunden - “ hinsichtlich der Erbringung von MRTUntersuchungen des Herzens nicht anders behandelt werden dürfe, als ein Radiologe.

Auf die Berufung der beklagten KV hob das Landessozialgericht (LSG) das Urteil des SG Berlin wieder auf. Nachdem auch das Bundessozialgericht (BSG) mit Urteil vom 02.04.2014 (Az.: B 6 KA 24/13 R) die Rechtsauffassung des LSG bestätigte, erhob der Kardiologe gegen diese beiden Urteile sowie gegen Qualitätssicherungsvereinbarungen KernspinV und MR-AngioV Verfassungsbeschwerde beim BVerfG. Darin rügte der Kardiologe insbesondere die Verletzung des in Art. 3 Abs. 1 GG verankerten Gleichheitsgrundsatzes. Weiterhin trug er zur Begründung vor, dass die neu geschaffene Zusatzweiterbildung „MRT - fachgebunden -“ ohne Anwendungsbereich bliebe, wenn fachgebundene MRT-Leistungen nicht auch durch entsprechend fortgebildete Fachärzte durchgeführt und abgerechnet werden dürften. Er sei zudem auf dem Teilgebiet der MRT-Untersuchungen des Herzens letztlich sogar besser qualifiziert als Radiologen.

3 Vereinbarung von Qualitätssicherungsmaßnahmen nach § 135 Abs. 2 SGB V zur MRAngiographie vom 01.10.2007.
Das BVerfG verwarf hingegen auch diese Verfassungsbeschwerde als unbegründet, da es keine Verletzung von Verfassungsrecht, insbesondere dem Gleichheitsgrundsatz aus Art. 3 Abs. 1 GG, erkennen konnte.

\section{Ausschluss anderer \\ Fachgruppen zur „Sicherung der Wirtschaftlichkeit“ gerechtfertigt}

Eine Verletzung von Art. 3 Abs. 1 GG durch den staatlichen Normgeber liegt dann vor, wenn eine Gruppe im Vergleich zu einer anderen Gruppe anders behandelt wird, obwohl zwischen beiden Gruppen keine Unterschiede von solcher Art und solchem Gewicht bestehen, dass sie die ungleiche Behandlung rechtfertigen könnten. Das BVerfG äußerte jedoch bereits Zweifel daran, ob die Gruppe der Kardiologen mit Zusatzweiterbildung „MRT - fachgebunden -“ und die Gruppe der Radiologen überhaupt vergleichbar seien. Einer abschließenden Entscheidung hinsichtlich der Frage der Vergleichsgruppenbildung bedurfte es letztlich nach Auffassung des BVerfG nicht; selbst bei einer Vergleichbarkeit der Gruppen sei eine Ungleichbehandlung von Radiologen und Kardiologen mit Zusatzweiterbildung „MRT - fachgebunden -“ zur „Sicherung der Wirtschaftlichkeit“ des Systems der gesetzlichen Krankenversicherung hinreichend gerechtfertigt.

Die Sicherung der Wirtschaftlichkeit im System der GKV war ein wesentlicher Gesichtspunkt für die Einführung des $§ 135$ Abs. 2 Satz 4 SGB V, der die Rechtsgrundlage für die Konzentration von MRT-Leistungen auf Radiologen durch die Regelungen der KernspinV und MR-AngioV darstellt. Dabei ging es dem Gesetzgeber insbesondere darum, die Trennung von kostspieliger Gerätediagnostik und Therapie zu ermöglichen, um keinen Anreiz dafür zu schaffen, dass sich Ärzte sog. Organfächer, z. B. Kardiologen, an sich selbst Patienten zur Erbringung radiologischer Leistungen überweisen. Insofern ist es im Interesse der Wirtschaftlichkeit sachgerecht, wenn die Normgeber an der strukturellen Trennung von Organ- und Methodenfächern festhalten. Außerdem so die Gesetzesbegründung - bewirke eine Arbeitsteilung im Sinne eines Mehraugenprinzips, dass die Diagnostik losgelöst von einem eventuellen wirtschaftlichen Interesse an einer daraufhin indizierten Therapie erfolgt. Damit sei sowohl der optimalen Patientenversorgung als auch dem sparsamen Einsatz der Leistungsressourcen gedient (vgl. BT-Drs. 15/1525, S. 124; vgl. auch BSG, Urteil Urteil vom 02.04.2014, Az.: B 6 KA 24/13 R).

Der Sicherung der Wirtschaftlichkeit diene letztlich - so das BVerfG - auch die Sicherung der Qualität, indem nicht nur ein bestimmtes Niveau der Versorgung, sondern auch der sparsamen Einsatz von Ressourcen gewährleistet werde. Nach §135 Abs. 2 Satz 4 SGB V, der die „Sicherung der Qualität“ zur Voraussetzung für den Erlass einschränkender Regelungen macht, müsse die Konzentration von Leistungen auf eine bestimmte Fachärztegruppe letztlich der Qualitätssteigerung dienen. Dies setze jedoch nicht voraus, dass die im Einzelfall bestmögliche Qualität angestrebt oder erreicht werde, sodass es nicht darauf ankomme, ob im Einzelfall - wie vorliegend durch den Beschwerdeführer behauptet eine noch bessere fachliche Qualifikation vorliegt. Vielmehr genüge es verfassungsrechtlichen Maßstäben, dass Radiologen, zu deren Fachgebiet im Kern die streitgegenständlichen MRT-Leistungen gehören, aufgrund ihrer Ausbildung hinreichende Gewähr für deren qualitative Durchführung bieten.

Eine hinreichende Qualität von MRT-Leistungen führe grundsätzlich auch zu geringeren finanziellen Belastungen der Krankenkassen, da die Gefahr unzureichender, zu wiederholender oder die Behandlung in eine falsche Richtung lenkender Untersuchungen sinke. Umgekehrt führe eine gesicherte Wirtschaftlichkeit grundsätzlich auch dazu, dass MRT-Leistungen hinreichend häufig und kostendeckend durchgeführt werden und dementsprechend die Qualität der Leistungserbringung gewährleistet sei. Gegen eine Differenzierung nach Facharztgruppen, wie es $\S 135$ Abs. 2 Satz 4 SGB V, KernSpinV und MR-AngioV vorsehen, bestünden, angesichts des in der BVerfG-Rechtsprechung wiederholt betonten Stellenwertes der Facharztausbildung für die Berufsausübung, keine verfassungsrechtlichen Bedenken. 


\section{„Sicherung der Qualität“ durch das Mehraugenprinzip}

Der Ausschluss der sogenannten Organfächer von der Erbringung von MRT-Leistungen ist nach Ansicht des BVerfG aber auch verhältnismäßig, da auf anderem Wege die Qualitätssicherung durch das Mehraugenprinzip nicht gewährleistet werden könne. Selbst durch eine Erweiterung des Überweisungsvorbehalts auf Fachärzte der Organfächer würden wirtschaftliche Fehlanreize nicht ebenso wirksam vermieden und nicht ausgeschlossen werden können, dass die Diagnostik unabhängig von der Therapie erfolgt. Denn eine Umgehung sei immer noch dadurch möglich, dass Fachärzte der Organfächer sich Patienten wechselseitig zur Diagnostik überweisen, was bei der allein Radiologen vorbehaltenen Durchführung von MRT-Leistungen nicht zu befürchten sei.

\section{Keine Teil-Untersuchungs-} und Abrechnungsteilbefugnis im GKV-Regelungskonzept vorgesehen

Darüber hinaus gehe die Beschränkung der Behandlungsbefugnis auf Radiologen einher mit der Abrechnungsbefugnis. Die Qualitätssicherungsvereinbarungen sähen keine nach Organen differenzierte Untersuchungs- und Abrechnungsteilbefugnis vor, sondern gingen von einer Gesamtbefugnis aufgrund der
Weiterbildungsinhalte der Fachgebiete aus. Die dafür erforderlichen umfassenden Kenntnisse würden nach dem Ausbildungsrecht jedoch allein Radiologen aufweisen. Die generelle Einbeziehung von Fachärzten, die über die Zusatzweiterbildung „MRT fachgebunden -“ verfügen, wäre daher zu weitgehend. Wegen der Vielzahl der Fachärzte wäre auch nicht nur eine untergeordnete Gruppe betroffen.

\section{Fazit}

Mit seinem dritten Beschluss zur Beschränkung von MRT-Leistungen im GKV-System hat das BVerfG klargestellt, dass diese auch nach der Einführung der Zusatzqualifikation „MRT - fachgebunden -“ in den Weiterbildungsordnungen der Länder verfassungsrechtlich gerechtfertigt ist. Der Gesetzgeber und die Vertragspartner der Bundesmantelverträge als untergesetzlicher Normgeber bleiben damit befugt, die Erbringung bestimmter Leistungen im GKV-System zur Sicherung der Wirtschaftlichkeit abweichend vom ärztlichen Weiterbildungsrecht zu regeln, solange ein Arzt nicht im Kernbereich seines Fachgebiets eingeschränkt wird. Dass dieser Aspekt im vertragsärztlichen Bereich über das Berufsrecht hinausgehende Beschränkungen erlaubt, hat das BVerfG in seinem Beschluss vom 01.02.2011 zur geringfügigen fachgebietsfremden Tätigkeit bereits betont (Az.: 1 BvR 2383/10). In seinen Ausführungen legt das BVerfG wie- derholt dar, dass sich die Sicherung der Qualität und die Sicherung der Wirtschaftlichkeit auch gegenseitig bedingen können und orientiert sich dabei an der Regelung des $§ 135$ Abs. 2 Satz 4 SGB V, der die Konzentration von medizinisch-technischen Leistungen auf bestimmte Fachgruppen nur zur Sicherung der Wirtschaftlichkeit und Qualität zulässt. Letztlich formuliert es dabei (gewollt oder ungewollt) für das GKV-System ein Verständnis von Qualität, die sich wiederum an ihrem Effekt auf die Wirtschaftlichkeit messen lassen muss.

Das Fachgebiet der Radiologie hat damit nochmals die Bestätigung erhalten, dass die Erbringung von MRT-Leistungen aus verfassungsrechtlichen Gründen nicht auf andere Facharztgruppen ausgeweitet werden muss.

Prof. Dr. Peter Wigge

Rechtsanwalt

Fachanwalt für Medizinrecht

Sophia K. Meinecke

Rechtsanwältin

Rechtsanwälte Wigge

Großer Burstah 42

20457 Hamburg

Telefon: (040) 3398705 - 90

Telefax: (040) 3398705 - 99

Internet: www.ra-wigge.de

E-Mail: kanzlei@ra-wigge.de 